\title{
Bronchiectasis Masquerading as Lung Metastasis in a Patient with Papillary Carcinoma Thyroid Identified by ${ }^{131}$ I Whole Body Scintigraphy
}

Padma $\mathbf{S}^{\star}$, Shanmuga Sundaram $\mathbf{P}$ and Firuz MD

Department of Nuclear Medicine \& Petct, Amrita Institute of Medical Sciences, Cochin-6802041, Kerala, India

*Corresponding author: Padma S, Clinical Professor, Department of Nuclear Medicine \& Petct, Amrita Institute of Medical Sciences, Cochin-6802041, Kerala, India, Tel: 91-484-2852001; Fax: 91-484-2852003; E-mail: padmas@aims.amrita.edu

Received date: Sep 01, 2015, Accepted date: Sep 23, 2015, Publication date: Sep 26, 2015

Copyright: @ 2015 Padma S, et al. This is an open-access article distributed under the terms of the Creative Commons Attribution License; which permits unrestricted use; distribution; and reproduction in any medium; provided the original author and source are credited.

\begin{abstract}
Differentiated thyroid carcinoma (DTC), arising from thyroid follicular epithelial cells, accounts for the vast majority of thyroid cancers. Despite being well-differentiated, papillary thyroid cancers may be overtly or minimally invasive. They may invade lymphatics but are less likely to invade blood vessels. lodine-131 ( $\left.{ }^{131} \mathrm{I}\right)$ is routinely used to image patients after thyroidectomy to assess the presence of residual thyroid tissue with or without metastasis. False positive ${ }^{131}$ I scans, showing the presence of ${ }^{131}$ I uptake in the absence of residual thyroid tissue or metastases can occur, although they are uncommon. Unless recognized as a false positive, ${ }^{131}$ I uptake may result in diagnostic error and lead to administration of an unnecessary higher therapeutic dose. We present a 55 year old female, histopathologically proven case of follicular variant of papillary carcinoma that underwent Total Thyroidectomy. Residual thyroid and whole body scan showed moderate residual thyroid tissue with abnormal ${ }^{131}$ I in right lung masquerading as right lung metastases. SPECTCT (single photon emission computed tomography - computed tomography) was incremental in localizing the site of ${ }^{131}$ I uptake to bronchiectatic changes. ${ }^{131}$ I diagnostic and post therapy scans are useful to risk stratify DTC patients' so that amount of high dose ${ }^{131} \mathrm{I}$ to be administered to patient can be estimated and judiciously used in their treatment.
\end{abstract}

Keywords: ${ }^{131}$ I whole body scan; Differentiated thyroid carcinoma; Papillary carcinoma of thyroid; Bronchiectasis

\section{Case History}

55 year old female, histopathologically proven case of follicular variant of papillary carcinoma underwent Total Thyroidectomy. Macroscopically tumour was $2.5 \times 0.7 \mathrm{~cm}$, margins are free with no lymphovascular emboli or perinodal spread. Residual thyroid and whole body ${ }^{131} \mathrm{I}$ scan was performed three weeks after the sugery. TSH was above $30 \mathrm{uIU} / \mathrm{ml}$ on the day of ${ }^{131} \mathrm{I}$ administration. Patient was also on iodine restricted diet and was off seafoods for three weeks prior to scan. Whole body and static anterior neck images showed moderate residual thyroid tissue with abnormal ${ }^{131} \mathrm{I}$ in right lung mimicking metastases (Figure 1). SPECT CT was performed which localized the site of abnormal ${ }^{131}$ I uptake to cystic bronchiectatic changes in right lung. On close interrogation patient suffered repeated upper respiratory tract infection. Stimulated serum Thyroglobulin (sensitive marker for identifying metastases) was $2.32 \mathrm{ng} / \mathrm{ml}$ confirming moderate residual thyroid tissue with no distant metastases.

Patient was orally ablated with 2220 Megabecqueral (i.e 60 millicuries, $\mathrm{mCi}$ ) of ${ }^{131} \mathrm{I}$ based on SPECTCT and Tg value. SPECTCT of thorax was incremental in localizing the ${ }^{131} \mathrm{I}$ uptake to bronchiectatic changes to right lung. Otherwise patient would require higher doses of $131 \mathrm{I}$ for therapy $(5550 \mathrm{Mbq}$, or $150 \mathrm{mCi}$ ) for lung metastases which is not only expensive but is an over treatment to this patient and produce higher radiation exposure. Post therapy ${ }^{131} \mathrm{I}$ scan performed (Figure 2) on day 5 revealed significant ${ }^{131}$ I uptake in thyroid bed as expected in a post therapy setting.

However there was new ${ }^{131}$ I uptake in left lung field which was against the previous finding of right lung involvement. SPECTCT of thorax in post therapy setting was performed which showed corresponding cystic bronchiectatic changes in bilateral lungs suggesting new left lung infection or inflammation. Thus ${ }^{131} \mathrm{I}$ diagnostic and post therapy scans are useful to risk stratify patients' so that amount of high dose ${ }^{131} \mathrm{I}$ to be administered to patient can be estimated and judiciously used in their treatment. It is also useful to identify any sites of occult metastases.

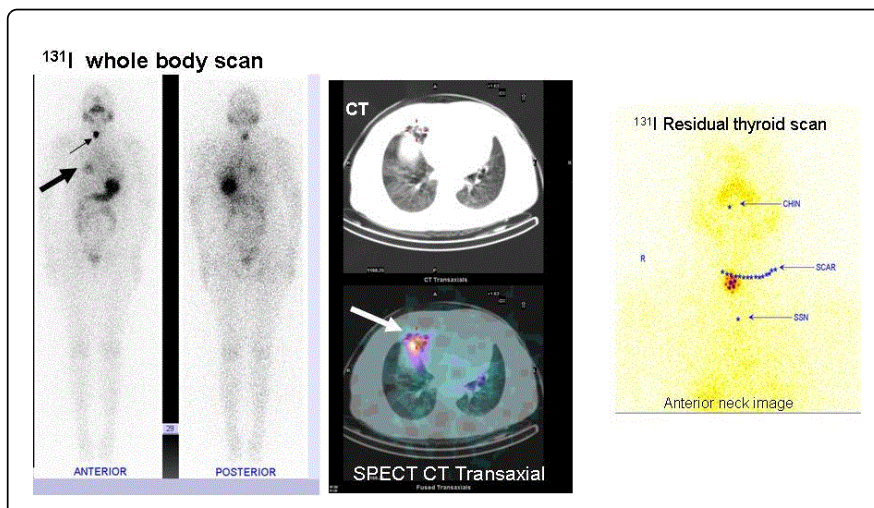

Figure 1: Residual thyroid and whole body ${ }^{131} \mathrm{I}$ scan showed moderate residual thyroid tissue (thin dotted arrow) with abnormal ${ }^{131} \mathrm{I}$ in right lung masquerading as metastases (thick arrow). Additional SPECT CT localized the site of abnormal ${ }^{131}$ I uptake to cystic bronchiectatic changes in right lung (thick arrow). 
Citation: Padma S, Sundaram PS, Firuz MD (2015) Bronchiectasis Masquerading as Lung Metastasis in a Patient with Papillary Carcinoma Thyroid Identified by ${ }^{131}$ I Whole Body Scintigraphy. J Nucl Med Radiat Ther 6: 252. doi:10.4172/2155-9619.1000252

Page 2 of 2

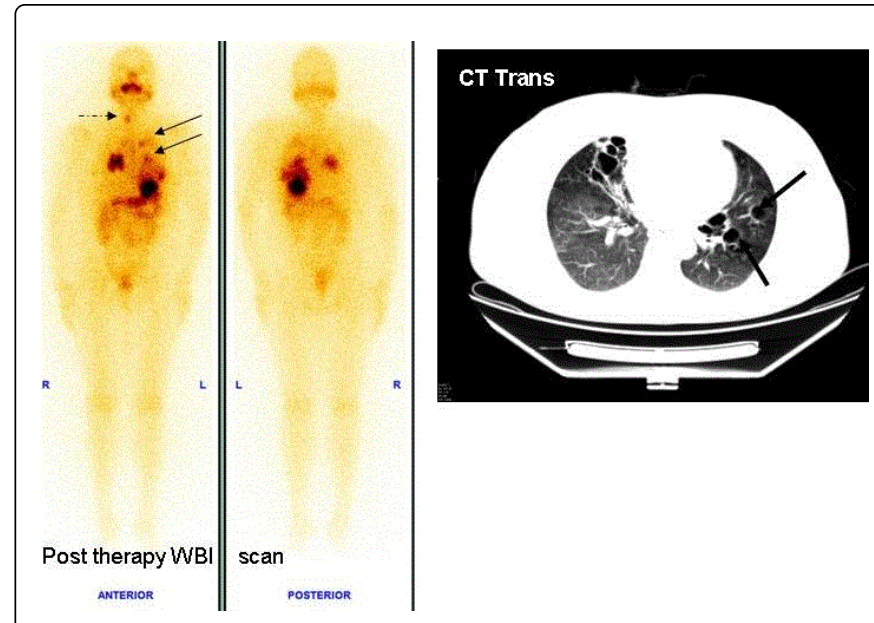

Figure 2: Post therapy ${ }^{131} \mathrm{I}$ scan performed on day 5 revealed significant ${ }^{131}$ Iuptake in thyroid bed as expected in a post therapy setting. However there was new ${ }^{131}$ I uptake in left lung field which was against the previous finding of right lung involvement. SPECTCT of thorax in post therapy setting showed corresponding cystic bronchiectatic changes in bilateral lungs suggesting new left lung infection/inflammation.

\section{Discussion}

${ }^{131}$ I is popularly termed the 'magic bullet' in the management of differentiated thyroid cancer for several years. SPECTCT in this setting is emerging as a useful tool in accurately localizing sites of pathological uptake and physiological mimics of disease, thus providing more accurate staging prognostic information for risk stratification, which in turn tailors management and follow-up regimes [1]. Thyrocytes retain the ability to trap iodine, and various isotopes of Iodine can be used both diagnostically and therapeutically in the management of DTC [2]. The correct interpretation of ${ }^{131} \mathrm{I}$ scans is critical in the appropriate management of patients with thyroid cancer. False positive findings do occur. A radioiodine scan showing abnormal uptake outside the thyroid bed must be studied carefully and alternative reasons for the finding must be considered. False-positive localizations of ${ }^{131} \mathrm{I}$ due to body secretions, pathologic exudate, activity in nonlactating breast, salivary gland inflammation, ovarian teratoma have been described [3]. The prognosis of PTC and follicular thyroid cancer are almost similar.

\section{Conclusion}

Our case, demonstrates an important pitfall in the interpretation of ${ }^{131}$ I scintigraphy in DTC patients. It calls attention to the fact that ${ }^{131} \mathrm{I}$ uptake may be related to various infectious and inflammatory processes, but infrequently with bronchiectasis, particularly with SPECTCT confirmation. In our patient incidentally there was progressive benign lung disease which misled diagnosis in two instances i.e. diagnostic and postherapy whole body ${ }^{131} \mathrm{I}$ scan thus representing a potential pitfall. So any focus of ${ }^{131} \mathrm{I}$ uptake must be looked upon carefully which will be worthwhile in the long run in deciding patient management.

\section{References}

1. Barwick TD, Dhawan RT, Lewington V (2012) Role of SPECT/CT in differentiated thyroid cancer. Nucl Med Commun 33:787-798.

2. Carlisle MR, Lu C, McDougall IR (2003) The interpretation of 131I scans in the evaluation of thyroid cancer, with an emphasis on false positive findings. Nucl Med Commun. 24: 715-735.

3. Rudoni S, Toubeau M, Mansuy S, Vaillant G, Verges B, et al. (1997) False positive scintigraphic images in the surveillance of differentiated thyroid cancers. Ann Endocrinol (Paris) 58: 399-407. 